\title{
Distributed sensing using stimulated Brillouin scattering : towards ultimate resolution.
}

\author{
A. Fellay, L. Thévenaz, M. Facchini, M. Niklès, P. Robert \\ EPFL, Swiss Federal Institute of Technology \\ Metrology Lab \\ $\mathrm{CH}-1015$ Lausanne, Switzerland \\ Alexandre.Fellay@met.de.epfl.ch
}

Stimulated Brillouin Scattering (SBS) shows the lowest threshold among all non-linear processes observed in optical fibers . It is also strongly dependent on local physical parameters of the fiber, since the scattered light experiences a frequency downshift $v_{B}$ with respect to the incident light proportional to the acoustic velocity within the fiber, this latter being function of temperature and strain. SBS is therefore naturally used to achieve distributed sensors measuring these quantities, and numerous contributions in this field have been presented in the past few years [ $1,2,3]$.

In this paper, we discuss the fundamental limitations of the SBS analysis as a distributed sensing method when the spatial resolution is in the meter range. We also present a novel experimental configuration that reaches the best performances achievable for this kind of sensors.

The basic configuration of a distributed Brillouin sensor is simple: a strong light pulse, hereafter called pump, is launched into the fiber. It crosses a weak CW lightwave, called signal or probe wave, that propagates in the backward direction. SBS occurs when pump and probe overlap, resulting in an amplification of the probe wave provided that the difference between the two frequencies lies within the Brillouin gain spectrum (BGS). This BGS shows a Lorentzian distribution centered on the Brillouin shift $v_{B}$ that is the quantity to determine. To obtain the BGS and thus determinev $v_{B}$, one simply measures the amplification of the Stokes wave while making a frequency scan. Let $g_{B}(v)$ be the gain spectrum; the net amplification of the signal wave after interaction with the pump pulse is given by

$$
I_{S}=I_{0} e^{g_{B}(v) I_{P} L},
$$

where the intensitics are $I_{o}$ for the incident signal wave, $I_{S}$ for the amplified signal wave and $I_{P}$ for the pump, respectively, and $L=T v_{g}$ is the equivalent pump pulse length, with $T$ the temporal width and $v_{g}$ the group velocity of the pump light.

The spatial resolution for distributed measurements is directly related to the pulse length $L$. As shown in equation 1, the amplification of the Stokes waves exponentially decreases when $L$ is getting shorter. This can be compensated by increasing proportionally the pump intensity, so that the product $I_{P} L$ remains constant, leaving a sufficient intensity for the measurement.

Figure 1 shows the experimental setup, based on a configuration developped in our laboratory [ 4 ]. Its main original feature is the presence of a single laser source that is modulated through an Mach-Zehnder electro-optic modulator (EOM) to generate both pump and probe lightwaves. This gives to the system an inherent stability, as far as frequency drifts of the laser are concerned. In addition, access to a single fibe end is required to perform the measurements, what is an obvious advantage in the field. On-site measurements have been so far performed using a $150 \mathrm{~mW}$ Nd:YAG laser at $1319 \mathrm{~nm}$, leading to a $3 \mathrm{~m}$ best resolution [ 5 ]. To improve this figure, it was necessary to boost the intensity of the pump wave, what can be ideally performed using an optical amplifier at $1550 \mathrm{~nm}$. 


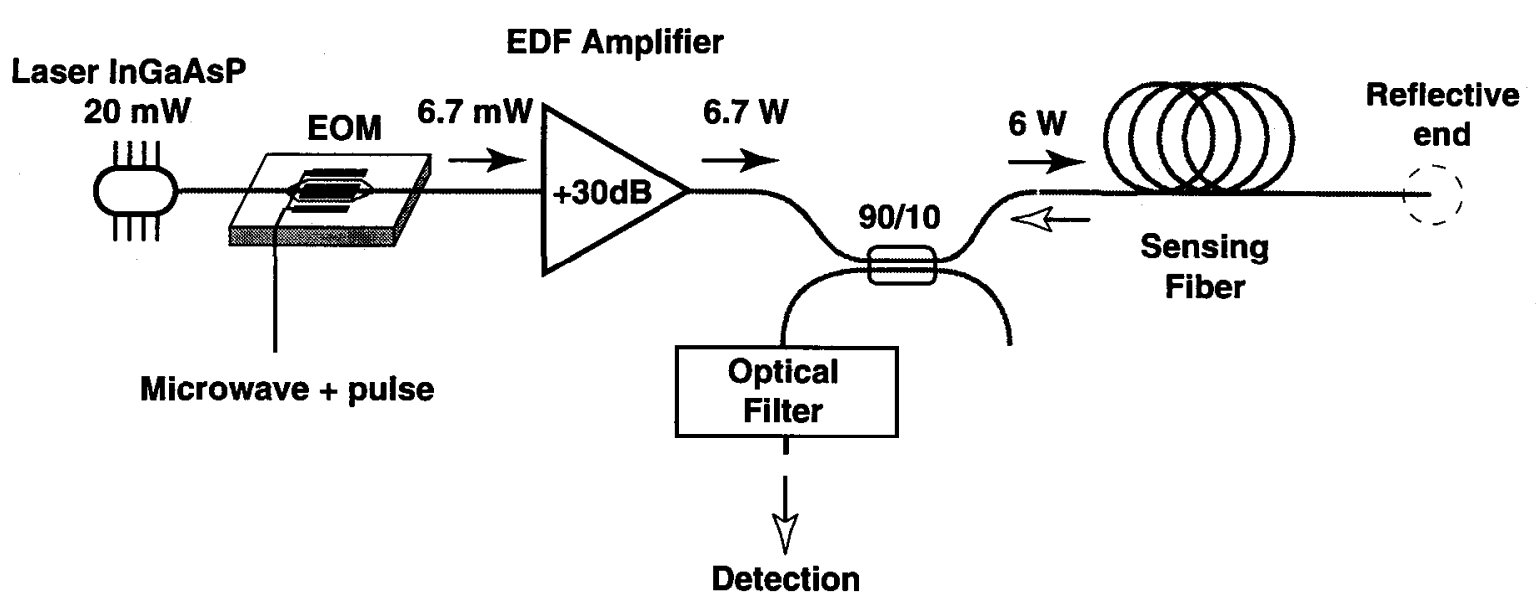

Fig. 1 Experimental setup of the EDFA-boosted Brillouin sensor. The light propagates forth and back through the sensing fiber and the optical filter removes the unwanted spectral components. The peak power of the pump pulse is indicated at different locations in the system.

The erbium-doped fiber amplifier (EDFA) has a typical small-signal gain of $30 \mathrm{~dB}$ and a saturation output power of about $15 \mathrm{dBm}$. In pulsed regime, even an input pulse with a several $\mathrm{mW}$ peak power may experience the small-signal gain, provided that the average output power does not exceed the saturation power. This can be realized by sufficiently decreasing the pulse duty cycle. Peak pump powers in the Watt range can thus be obtained. Minimal absorption loss is a further advantage of the $1550 \mathrm{~nm}$ transmission window, making a $100 \mathrm{~km}$ sensing range possible.

The first measurements performed using the novel configuration immediately show a significantly improved spatial resolution. A $80 \mathrm{~cm}$ fiber segment experiencing a $1.5 \%$ elongation can be clearly identified, as shown in Figure 2.

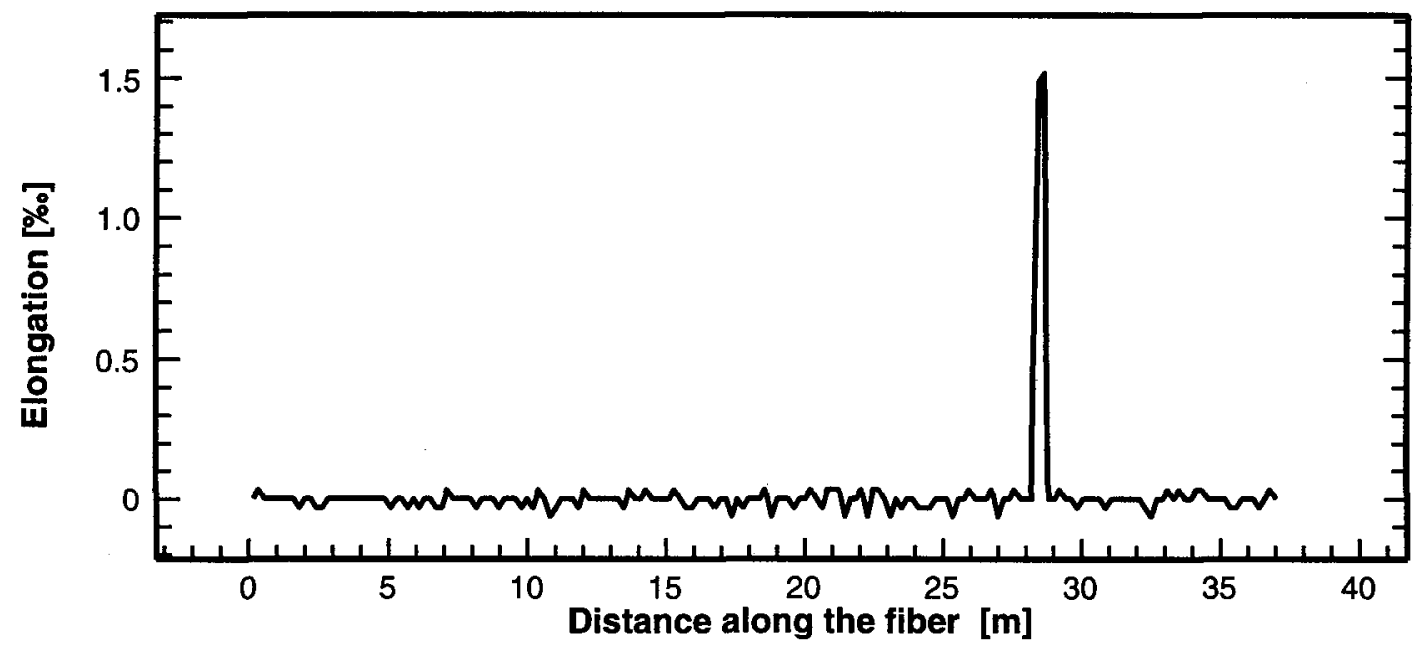

Fig. 2 Distributed measurement along a single mode fiber using the EDFA-boosted Brillouin sensor. A 80-cm fiber segment stretched using a $155 \mathrm{~g}$ weight is clearly identified, resulting in a $1.52 \%$ elongation.

The measurements shown in Figure 2 have been performed using a $1 \mathrm{~m}$ wide pulse. A significative improvement of this resolution meets with two obstacles. The first problem is trivial : the pulse narrowing results in an exponential decrease of the measured signal, as a consequence of equation 1 . This could be compensated to a limited extent by increasing the detector sensitivity and by using a more sophisticated data processing. 


\section{6 / OWD3-3}

The second limitation is more fundamental: it results from the pulsed nature of the pump wave, and determines the ultimate resolution of the Brillouin sensor. In Equation 1, it was assumed that the gain spectrum $g_{\mathrm{B}}(v)$ does not depend on the length $L$ of the pump pulse. This is no longer true for short pulses. The pulse narrowing induces a broadening ot its spectrum, so that the effective $B G S \overline{g_{B}}(v)$ experienced by the signal wave is given by the convolution between the original $g_{B}(v)$ and the spectrum of the pump pulse. Assuming a perfectly rectangular pulse of width $L$, this convolution yields the following expression :

$$
\overline{g_{B}}(v)=2 e^{-\pi \Delta v_{B} L}\left\{\cosh \left(\pi \Delta v_{B} L\right)-\cos \left(2 \pi\left(v-v_{B}\right) L\right)\right\} g_{B}(v)
$$

$\overline{g_{B}}(v)$ must replace the intrinsic Lorentzian spectrum $g_{B}(v)$ in equation 1 to calculate the actual amplification of the probe signal. Figure 3 shows the measured effective BGS for three pulse widths. In total agreement with equation 2, the curves spread and become broader for shorter pulses, resulting in a dramatic decrease of the peak gain, and of the measured signal consequently. In addition, this effect is detrimental for the frequency resolution of the measurements, the central frequency of such a spread distribution being determined with less accuracy.

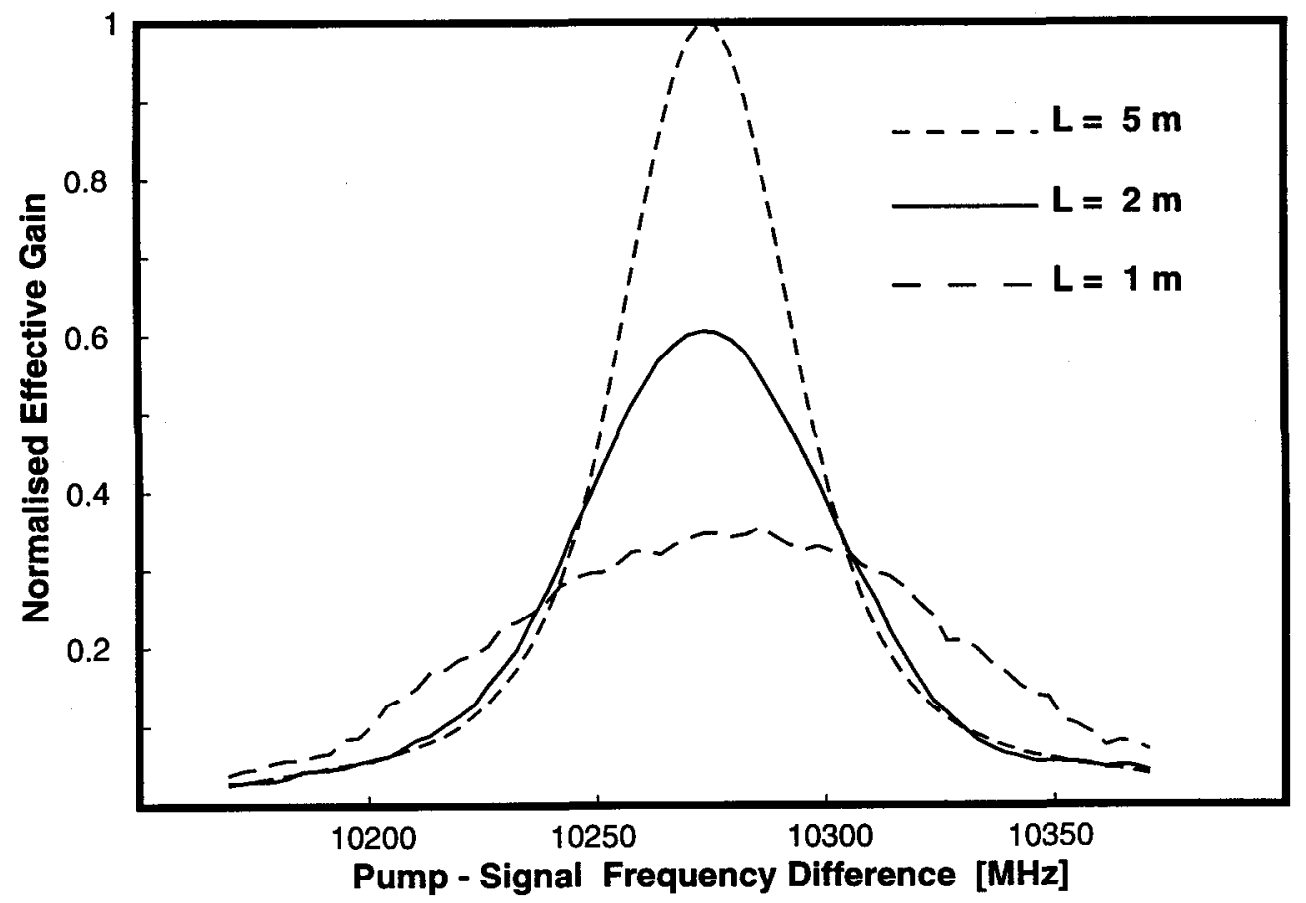

Fig. 3 Effective Brillouin gain spectrum for different pulse lengths $L$. The vertical full scale corresponds to th maximum of the Brillouin gain for continuous pump wave. The curves speading for short pulses results from the convolution of the pulse spectrum with the intrinsic Brillouin gain spectrum.

It is possible to estimate the ultimate resolution of a distributed Brillouin sensor as a function of the fiber length : it is the minimal pulse length giving a sufficient amplification, say $2 \%$, when the spreading of the gain spectrum and the effect of absorption are taken into account. With our system specifications, the calculation yields the relation shown in Figure 4. The most remarkable feature shown on this graph is the maintained resolution over a long distance : it remains below 3 meters over $50 \mathrm{~km}$, as a consequence of the low loss at $1550 \mathrm{~nm}$. On the other hand, the optimal resolution for very short fibers is slightly less than $1 \mathrm{~m}$. This kind of sensors is thus definitely dedicated for long range measurements with meter resolution and is not suitable for a centimeter resolution. 


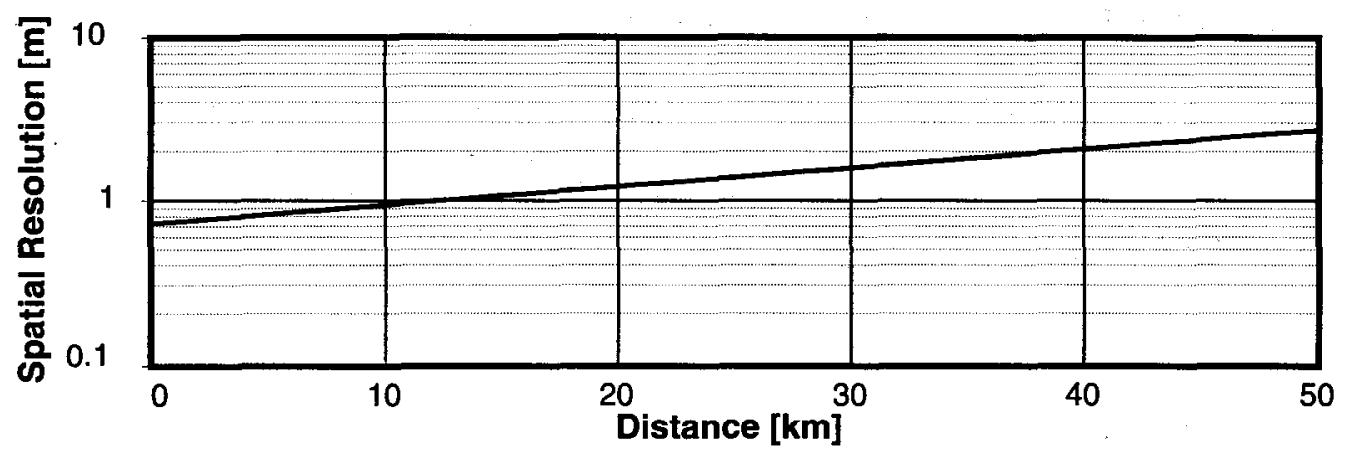

Fig. 4 Spatial resolution leaving a sufficient umplification (2\%) vs distance along the fiber. The pump power is $W$, the attenuation $0.22 \mathrm{~dB} / \mathrm{km}$ and the average linear Brillouin gain $1 \cdot 1 b^{l} \mathrm{~W} \cdot \mathrm{m}$.

Due to the basic physical features of the measuring process, the spatial resolution of SBS analysis is limited to a few tens of centimeter. The EDFA-boosted sensor we have presented here has actually reached this limit. In spite of this rather strong limitation, the applications of Brillouin sensing remain numerous : the figure of merit shown in Figure 4 indicates in particular that the EDFA-boosted Brillouin sensor is very competitive when a resolution in the meter range is needed over a considerable distance. Some typical applications are the detection of defects in telecom fibers, the monitoring of deformations in large-sized concrete structures like tunnels or dams, and the centralized temperature survey of building at a city scale.

\section{References}

[ 1 ] T. Kurashima, T. Horiguchi, M. Tateda, Optics Lett. 15, p. 1038 (1990)

[ 2] X. Bao et al. ,J. Lightwave Technol. 13, p. 1340 (1995)

[ 3 ] M. Niklès, L. Thévenaz, P. Robert, Proceedings OFS'94, p. 138 (1994)

[ 4 ] M. Niklès, L. Thévenaz, P. Robert, Optics Lett. 21, p. 758 (1996)

[ 5 ] M. Niklès, L. Thévenaz, P. Salina, P. Robert, Technical Digest OFMC'96, NIST Special

Publication 905, p 111 (1996) 